
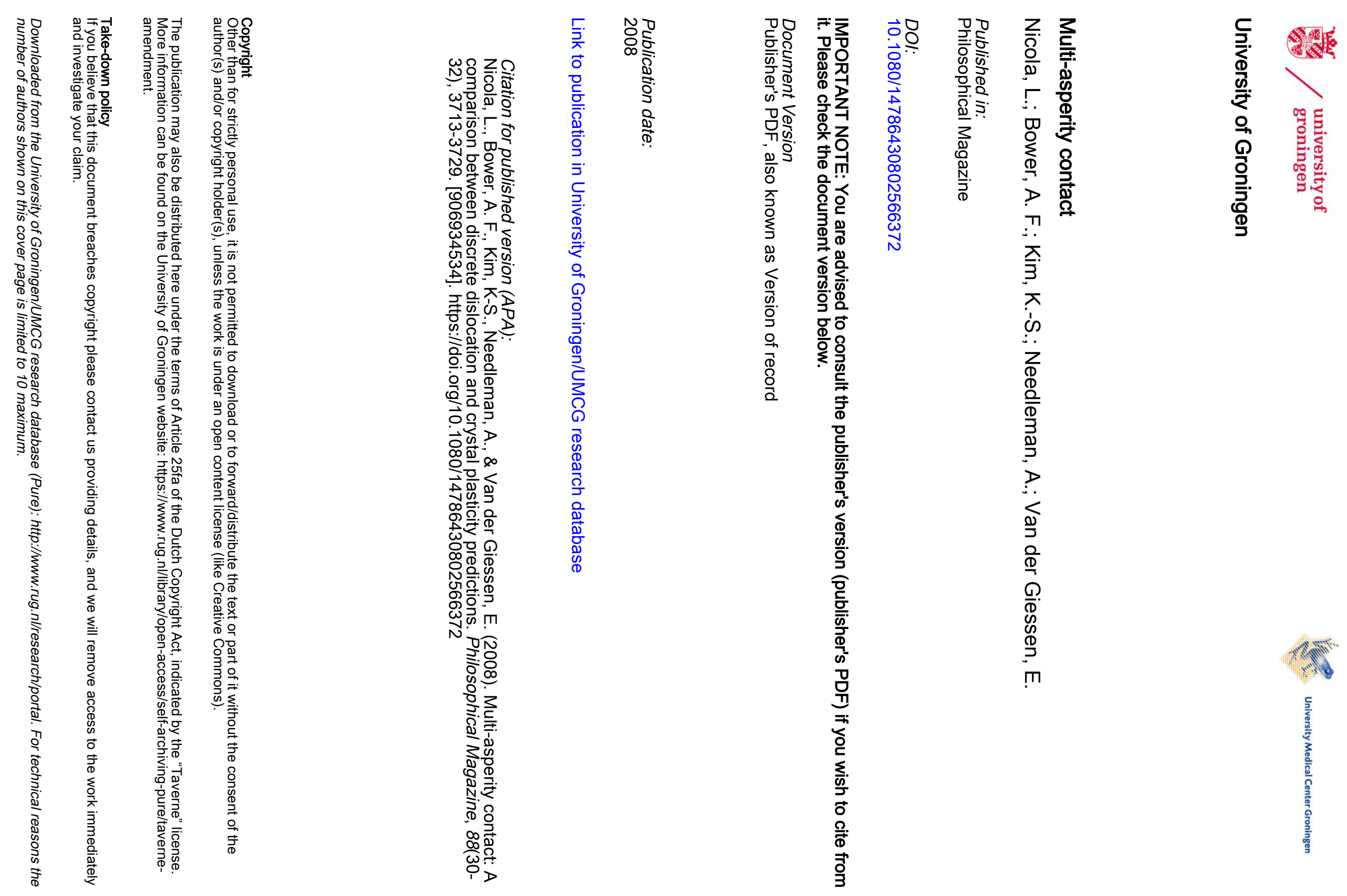


\title{
Multi-asperity contact: A comparison between discrete dislocation and crystal plasticity predictions
}

\author{
L. Nicola ${ }^{a} *$, A.F. Bower ${ }^{a}$, K.-S. Kim ${ }^{\text {a }}$, A. Needleman ${ }^{\text {a }}$ and E. Van der Giessen ${ }^{\text {c }}$ \\ ${ }^{a}$ Division of Engineering, Brown University, Providence, USA; ${ }^{b}$ Department of Materials \\ Science and Engineering, Delft University of Technology, Delft, The Netherlands; ' Department \\ of Applied Physics, University of Groningen, Groningen, The Netherlands
}

(Received 10 January 2008; final version received 16 October 2008)

\begin{abstract}
Plane strain indentation of single crystals by a periodic array of flat rigid contacts is analyzed. The calculations are carried out, with the mechanical response of the crystal characterized by conventional continuum crystal plasticity or by discrete dislocation plasticity. The properties used in the conventional crystal plasticity description are chosen so that both theories give essentially the same response in uniform plane strain compression. The indentation predictions are then compared, focusing in particular on the effect of contact size and spacing. The limiting cases of frictionless contacts and of perfectly sticking contacts are analyzed. Conventional continuum plasticity predicts a size-independent response. Unless the contact spacing to size ratio is very small, the predicted deformation mode under the contacts is a wedging mechanism of the type described by slip line theory, which is only weakly sensitive to friction conditions. For the micron scale contacts analyzed, discrete dislocation plasticity predicts a response that depends on the contact size as well as on the contact spacing to size ratio. When contacts are spaced sufficiently far apart, discrete dislocation plasticity predicts that the deformation is localized beneath the contacts, whereas for more closely spaced contacts, deformation occurs by shear bands extending relatively far into the crystal. Unless the contacts are sufficiently close together so that the response is essentially one of plane strain compression, the mean contact pressure predicted by discrete dislocation plasticity is substantially greater than that predicted by conventional continuum crystal plasticity and is more sensitive to the friction conditions.
\end{abstract}

Keywords: contact; plasticity; dislocation; size effect

\section{Introduction}

Contact between rough surfaces can play a key role in a variety of phenomena of both scientific and technological interest, for example, friction and wear. One of the major sources of dissipation in machines is the plastic deformation that occurs in the vicinity of the contact surface, which can control the ensuing response. Hence, there is much interest in the development of models that can predict the local stress and deformation state near the asperities of rough surfaces. Asperity sizes typically range from a few nanometers to

*Corresponding author. Email: L.Nicola@tudelft.nl 
several microns. This is precisely the range in which plastic deformation of crystalline solids is known to be size dependent and thus not adequately described by conventional continuum plasticity theory. Discrete dislocation plasticity simulations have proven to be capable of capturing size-dependent plasticity in various situations, including (sub)micronindentation with single indenters [1-4].

Recent models of contact between elastic-plastic rough surfaces have shown that interactions between neighboring asperity contacts play a critical role in determining the true area of contact between the surfaces [5-7]. Asperity interactions increase the apparent hardness of the solid, and so reduce the true contact area. These predictions are based on classical plasticity theory, and so neglect any additional strengthening due to indentation size effects. Size effects for isolated contacts have been extensively studied both computationally [1-3] and experimentally [8,9]. Kim and co-workers have recently undertaken experimental studies on the evolution of multi-asperity contact [10]. In [10], the rough surface of a gold single crystal was compressed by mica under dry and lubricated contact. The deformed surface deviates from classical plasticity predictions, especially under dry contact loading. The extent to which the deviation from the predictions of conventional continuum plasticity can be attributed to the size dependent response of single asperities, as in [9], or to asperity interactions, remains to be determined. On the other hand, the relevance of asperity interactions in forming contact is widely recognized and has been recently emphasized, among others, by Zhao and Chang [11] and Ciavarella [12]. The current paper aims at investigating the influence of size effects on asperity interactions.

We recently carried out a study of asperity contact effects, in [13], that focused on the competing roles of surface and subsurface dislocation sources on the plastic flow under a rough surface. These discrete dislocation plasticity results showed that nucleation from subsurface dislocation sources could account for the apparently paradoxical experimental observation [14] of a tensile residual stress near the contact surface. Conventional crystal plasticity and discrete dislocation plasticity with bulk dislocation sources predict a corresponding compressive residual stress. This study also showed that the plastic zone under the contacts predicted by discrete dislocation plasticity was much larger than that occurring, according to conventional crystal plasticity.

In the present paper, we address in detail the influence of asperity interactions on hardness, ignoring surface nucleation. To this end we carry out simulations for values of contact size to contact spacing, varying by nearly an order of magnitude, from $1 / 1.5$ to $1 / 12$. The predictions of discrete dislocation plasticity are compared with those of conventional continuum plasticity for the mean indentation pressure versus indentation depth response, as well as for the stress and deformation states. Such a comparison aims at revealing limits on the accuracy of conventional crystal plasticity analyses, as well as at providing an understanding of the origin of the discrepancy with discrete dislocation plasticity predictions. Also, in contrast to conventional continuum crystal plasticity, discrete dislocation plasticity predicts the occurrence of two distinct modes of deformation depending on the contact fraction, defined as the ratio between contact size and contact spacing, and the size of the asperities.

Discrete dislocation plasticity is a method of solving problems, where plastic flow is represented in terms of the collective motion of discrete dislocations. The formulation was presented by Van der Giessen and Needleman in [15] and has been used to solve a variety of two and three dimensional boundary value problems, e.g. [16-21]. In the calculations 
here, a two dimensional discrete dislocation plasticity formulation is used with the dislocations all of edge character and modeled as line singularities in an isotropic linear elastic solid. Constitutive rules are specified for dislocation nucleation, dislocation glide, interaction with obstacles and dislocation annihilation. There are aspects of dislocation plasticity that cannot be modeled within the two-dimensional framework used here, such as cross-slip and the dynamic evolution of dislocation sources and obstacles, that play an important role in the evolution of work hardening. Although improvements have been proposed [22], the range of phenomena that the framework used here can model is limited. However, there are circumstances involving plastic deformation in small volumes, where long-range elastic dislocation interactions dominate, which permits a wide range of complex phenomena involving plastic deformation to be represented qualitatively, e.g. $[4,17,23]$, and, to a remarkable extent even quantitatively, e.g. [16,24]. In such circumstances, the dislocation-dislocation interactions that are so important for work hardening do not play a major role. Indeed, the experiments in [10] show that the main mechanism responsible for the evolution of the surface roughness under compression is easy glide, occurring along either the $\langle 100\rangle$ or $\langle 110\rangle$ directions in the gold crystal.

Plane strain indentation problems are analyzed, with the analyses carried out within a small deformation gradient context. The material properties used in the continuum crystal plasticity calculations are chosen so that the plane strain compression (or tension) response is nearly the same as that obtained from a corresponding discrete dislocation plasticity calculation.

\section{Formulation}

\subsection{Boundary value problem}

The boundary value problem analyzed is the same as in [13] and, for completeness, is briefly specified here. As sketched in Figure 1, the indentation of a planar single crystal of height $h$ is subject to indentation by a rigid indenter with a rectangular wave profile with period $w$. The contact width is $a$ and plane strain conditions are assumed. The material is assumed to possess the same periodicity as the indenter, and attention is therefore confined

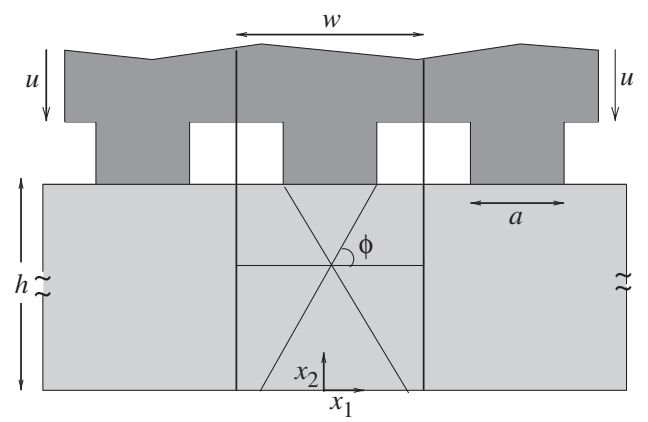

Figure 1. Two-dimensional model of a single crystal indented by a rough surface with flat contacts of width $a$ at a center-to-center spacing $w$. Calculations are carried out for a periodic unit cell of width $w$. 
to a unit cell $-w / 2 \leq x_{1} \leq w / 2$. Indentation is imposed by applying a constant displacement rate of the rigid contacts, $\dot{u}$, in the negative $x_{2}$-direction. We analyze two limiting cases: (i) frictionless contacts and (ii) perfectly sticking contacts.

The computations are carried out for a unit cell and the contact with the crystal occurs on $-a / 2 \leq x_{1} \leq a / 2$. Indentation is prescribed by specifying the normal displacement rate along the contact surface,

$$
\dot{u}_{2}\left(x_{1}, h\right)=-\dot{u}, \quad-\frac{a}{2} \leq x_{1} \leq \frac{a}{2},
$$

with one of the following two tangential conditions prescribed between the contact and the crystal,

(i) Frictionless: $\sigma_{12}\left(x_{1}, h\right)=0, \quad-\frac{a}{2} \leq x_{1} \leq \frac{a}{2}$,

(ii) Perfectly sticking: $\quad u_{1}\left(x_{1}, h\right)=0, \quad-\frac{a}{2} \leq x_{1} \leq \frac{a}{2}$.

The remainder of the top surface of the unit cell (see Figure 1) is traction-free; i.e.

$$
\sigma_{12}\left(x_{1}, h\right)=\sigma_{22}\left(x_{1}, h\right)=0, \quad \pm x_{1} \in\left[\frac{a}{2}, \frac{w}{2}\right] .
$$

The boundary conditions along the bottom of the unit cell, $x_{2}=0$, are taken to be

$$
u_{2}\left(x_{1}, 0\right)=0, \quad \sigma_{12}\left(x_{1}, 0\right)=0 .
$$

The resulting indentation force is computed from the normal traction along the contact surface as

$$
f=\int_{-a / 2}^{a / 2} \sigma_{22} \mathrm{~d} x_{1} .
$$

Periodic boundary conditions are imposed on the sides of the unit cell by requiring

$$
\begin{gathered}
u_{1}\left(\frac{w}{2}, x_{2}\right)=u_{1}\left(-\frac{w}{2}, x_{2}\right)+V \\
u_{2}\left(-\frac{w}{2}, x_{2}\right)=u_{2}\left(\frac{w}{2}, x_{2}\right) .
\end{gathered}
$$

In addition, we impose

$$
u_{1}\left(-\frac{w}{2}, 0\right)=0
$$

which, for frictionless contacts, prevents rigid body translation parallel to the $x_{1}$-axis.

The value of the uniform expansion $V$ in Equation (7) depends on the friction between the contacting surfaces. For perfectly sticking contacts, the unit cell cannot expand or contract because the indenter is rigid so that $V=0$. On the other hand, for frictionless contacts, the unit cell can expand in the $x_{1}$-direction. We assume that there is no remotely imposed loading parallel to the surface, so the resultant $x_{1}$-component of force acting 
on any plane $x_{1}=$ constant vanishes. Hence, in the absense of friction, the value of $V$ is determined from the condition

$$
\frac{1}{h} \int_{0}^{h} \sigma_{11}\left(x_{1}, x_{2}\right) \mathrm{d} x_{2}=0
$$

\subsection{Discrete dislocation plasticity}

Following [15], the solution to the boundary value problem, formulated in Section 2.1, is obtained by decomposing all fields into a ( )-field part governed by the singular fields of the individual dislocations in infinite space, and an image part $\left(^{\wedge}\right)$ that corrects for the boundary conditions. The ( )-fields for a given distribution of dislocations that is periodic in the $x_{1}$-direction are known analytically [25]. The ( ${ }^{\wedge}$ )-field is obtained as the solution of an elasticity boundary value problem for the unit cell, with boundary conditions affected by the current dislocation distribution, which we solve by the finite element method. Since the ( )-fields used satisfy periodicity in the $x_{1}$-direction, the periodicity condition Equation (8) needs to be imposed on the $\left(^{\wedge}\right)$-field. This condition is enforced by a penalty function approach.

For frictionless contacts, an additional boundary condition is imposed for the discrete dislocation solutions, namely,

$$
u_{1}(0, h)=u_{1}(0,0)
$$

This condition prevents overall shearing of the unit cell, which can occur in some calculations due to the lack of symmetry of the dislocation distribution. This condition is not required in the crystal plasticity calculations because they maintain symmetry about $x_{1}=0$.

The connection between the stress state in the body and the evolution of the dislocation structure is given through a set of constitutive equations, similar to the ones proposed in [26] and used previously in [13] as well as in related investigations, [3,4,27]. These rules control the nucleation, glide, annihilation of dislocations, as well as their pinning at obstacles.

Nucleation occurs by activation of Frank-Read sources, which are taken to be initially present in the material. The dislocation sources are positioned on the slip planes and their density is taken to be constant during the simulation. A critical shear stress must act on a source to make it operate by bowing out the Frank-Read segment, and form a new dislocation loop. Three parameters are associated with each source: a critical strength necessary to create the new dislocation loop, the critical time $t_{\text {nuc }}$ required for its formation and the diameter of the loop at nucleation, $L_{\text {nuc }}$.

After nucleation, the glide velocity $v^{I}$ of the $I$ th dislocation is proportional to the Peach-Koehler force $f^{I}$, according to $f^{I}=B v^{I}$ with $B$ the drag coefficient.

Dislocation glide can be stopped by the presence on the slip planes of point obstacles, each characterized by a critical strength, $\tau_{\text {obs }}$. As long as the resolved shear stress acting on the pinned dislocation is lower than the obstacle strength, the dislocation stays pinned at the obstacle. 
Dislocation annihilation occurs when opposite-signed dislocations meet. This is modeled by removing dislocations of opposite sign from the simulation when they are on the same slip plane closer to each other than the critical material-dependent distance $L_{\text {ann }}$. Dislocations can exit the crystal through the top $\left(x_{2}=h\right)$ free surface, $\pm x_{1} \in[a / 2, w / 2]$. Along the parts of the surface that are in contact with the rigid indenter, dislocations do not cross the interface. No special algorithm is required for this; it is ensured by the incompatibility of the dislocation displacement fields with the prescribed displacement boundary conditions in Equations (1)-(4).

\subsection{Crystal plasticity}

The formulation and numerical implementation of conventional (size-independent) viscoplastic continuum crystal plasticity model follow that in [28]. However, here, attention is confined to small displacement gradients. The total strain rate is written as the sum of an elastic part and a viscoplastic part

$$
\dot{\varepsilon}_{i j}=\dot{\varepsilon}_{i j}^{\mathrm{e}}+\dot{\varepsilon}_{i j}^{\mathrm{p}} .
$$

The plastic part of the strain rate is given by

$$
\dot{\varepsilon}_{i j}{ }^{\mathrm{p}}=\sum_{\alpha} \dot{\gamma}_{\alpha} \mu_{i j}^{(\alpha)}, \quad \mu_{i j}^{(\alpha)}=\frac{1}{2}\left[s_{i}^{(\alpha)} m_{j}^{(\alpha)}+s_{j}^{(\alpha)} m_{i}^{(\alpha)}\right],
$$

where $m_{i}^{(\alpha)}$ and $s_{i}^{(\alpha)}$ are, respectively, the components of the slip plane normal, and the slip direction of slip systems $\alpha$ and $\dot{\gamma}_{\alpha}$ is the intrinsic slip rate. The elastic part of the strain rate is specified by Hooke's law,

$$
\dot{\sigma}_{i j}=\mathcal{L}_{i j k l} \dot{\varepsilon}_{k l}^{\mathrm{e}} .
$$

Here, $\mathcal{L}_{i j k l}$ is the tensor of isotropic elastic moduli with shear modulus $\mu$ and Poisson's ratio $v$.

The slip rate is given by the power law relation

$$
\dot{\gamma}_{\alpha}=\dot{\gamma}_{0} \frac{\tau_{\alpha}}{g_{\alpha}}\left|\frac{\tau_{\alpha}}{g_{\alpha}}\right|^{\left(\frac{1}{m}-1\right)},
$$

with $\tau_{\alpha}=m_{i}^{(\alpha)} \sigma_{i j} s_{j}^{(\alpha)}$ the resolved shear stress on slip system $\alpha ; \dot{\gamma}_{0}$ is a reference slip rate, $m$ is the strain rate sensitivity exponent and $g_{\alpha}$ is the hardness of slip system $\alpha$, which has initial value $\tau_{0}$ for all $\alpha$ and evolves according to

$$
\dot{g}_{\alpha}=h_{0} \sum_{\alpha}\left|\dot{\gamma}^{(\alpha)}\right| .
$$

The finite element discretization uses a mesh of rectangular elements, each consisting of four triangles to avoid locking problems associated with near incompressibility.

\section{Choice of parameters}

The elastic constants are taken to be representative of an FCC metal: the shear modulus is taken to be $\mu=26 \mathrm{GPa}$ and Poisson's ratio $\nu=0.33$ as in aluminum. The three slip systems 
have slip plane orientations: $\phi^{(1)}=0^{\circ} ; \phi^{(2)}=60^{\circ} ; \phi^{(3)}=120^{\circ}$ and have the same initial strength $\tau_{0}=20 \mathrm{MPa}$. For a fair comparison between the two models, the crystal plasticity parameters are taken such that in plane strain compression the two responses are similar. Discrete dislocation plasticity simulations of (plane strain) compression or tension of single crystals with the parameters as specified below reveal essentially no hardening, yet; in order to avoid numerical difficulties associated with non-hardening behavior in the continuum plasticity calculations, a small hardening rate, $h_{0} / \mu=2.5 \times 10^{-6}$, is specified. A reference slip rate of $\dot{\gamma}_{0}=2 \cdot 10^{3}$ is used and the rate sensitivity exponent in Equation (15) is taken to be $m=0.005$, which is a representative value for most FCC metals at room temperature, and which corresponds to essential rate independent behavior.

In the discrete dislocation calculations, the crystal contains a set of discrete slip planes at $\left(0^{\circ}, 60^{\circ}, 120^{\circ}\right)$ spaced at $200 b$, the magnitude of the Burgers vector being $b=0.25 \mathrm{~nm}$. Initially, the crystal is dislocation free, but contains a density $\rho_{\text {nuc }}=30 / \mu \mathrm{m}^{2}$ of dislocation sources. The strength of the sources is taken randomly from a Gaussian distribution with mean strength $\bar{\tau}_{\text {nuc }}=50 \mathrm{MPa}$ and standard deviation of $10 \mathrm{MPa}$. The critical time for nucleation is $t_{\text {nuc }}=0.1 \mathrm{~ns}$. The sources are located at random positions on randomly selected slip planes; some of the slip planes contain no source and are therefore inactive during the simulation. A density $\rho_{\text {obs }}=30 / \mu \mathrm{m}^{2}$ of point obstacles with strength $\tau_{\mathrm{obs}}=150 \mathrm{MPa}$ is taken to be present throughout the crystal. Dislocation obstacles are positioned only on slip planes containing at least one dislocation source. The annihilation distance, $L_{\mathrm{ann}}$, is taken to be $6 b$.

Both in the continuum crystal plasticity and in the discrete dislocation plasticity calculations, the loading is applied by prescribing a constant displacement rate $\dot{u}=4 \times 10^{4} \mu \mathrm{m} / \mathrm{s}$ in Equation (1).

\section{Results}

\subsection{Evolution of the mean contact pressure}

Calculations are carried out for crystals with contact fractions $a / w=1 / 1.5,1 / 3,1 / 6,1 / 9$ and $1 / 12$. The contact fraction is varied by changing the period $w$ and keeping the contact size fixed at $a=1 \mu \mathrm{m}$, while $h=50 \mu \mathrm{m}$.

Figure 2 shows results for the variation of the mean contact pressure, $P_{\mathrm{m}}=f / a$, with indentation depth $u$ for both frictionless and perfectly sticking contacts. The solid curves show the predictions of discrete dislocation plasticity while the corresponding dashed curves show the predictions of continuum crystal plasticity. Note that, as with all plane contact problems, the elastic compliance of the contact is sensitive to the remote boundary conditions, and would increase in proportion to $\log (h / a)$ as $h / a \rightarrow \infty$. However, the values of the mean contact pressure $P_{\mathrm{m}}$ required to initiate plastic deformation or plastic collapse in continuum plasticity (when it occurs) are not sensitive to the cell height, although the corresponding indentation depth values are.

For frictionless contacts (Figure 2a), there is good agreement between the crystal plasticity and the discrete dislocation plasticity results when the contacts are closely spaced; i.e. for contact fractions of $a / w=1 / 1.5$ and $a / w=1 / 3$ (recall that the crystal plasticity material parameters were chosen to give the same plane strain compression response as given by discrete dislocation plasticity). However, the predictions of these two 

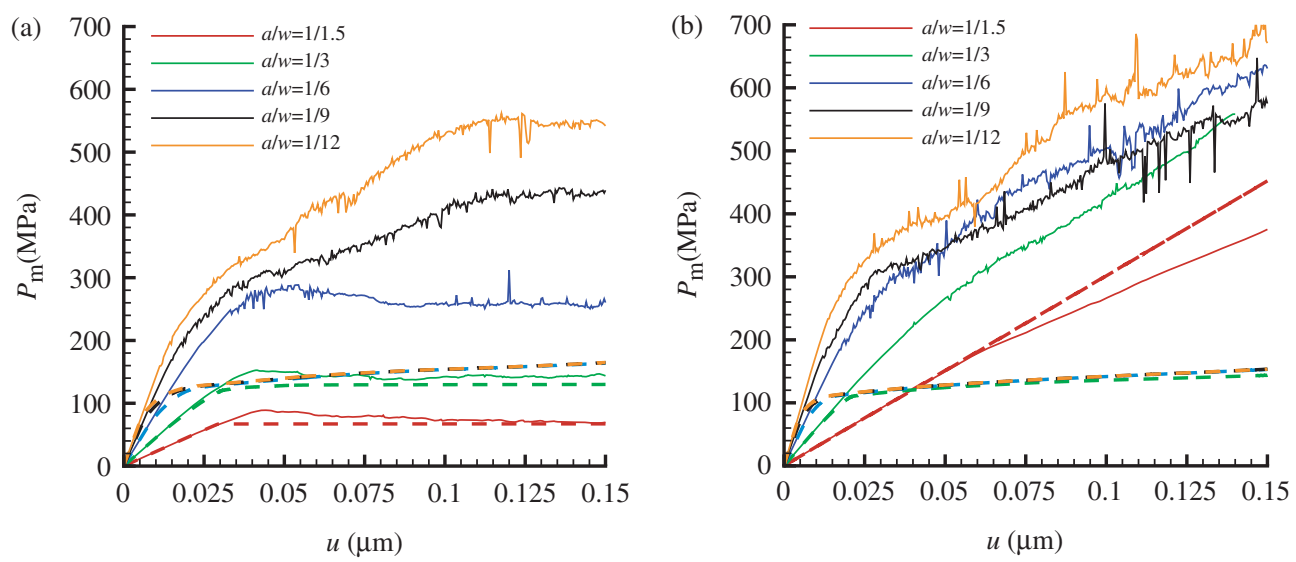

Figure 2. Mean contact pressure, $P_{\mathrm{m}}$, versus indentation depth, $u$, for crystals with a contact size $a=1 \mu \mathrm{m}$ and various contact fractions for (a) frictionless and (b) perfectly sticking contacts. Dashed lines indicate continuum crystal plasticity simulations, solid lines discrete dislocation plasticity simulations.

material characterizations differ substantially when the contacts are widely spaced (i.e. for $a / w=1 / 9$ and $a / w=1 / 12$ ) with discrete dislocation plasticity predicting a harder response.

The discrete dislocation plasticity calculations show a transition between the behavior for a small contact fraction and that for a large contact fraction. For $a / w<1 / 6$, yielding, defined as the mean pressure at a $0.002 \mu \mathrm{m}$ displacement offset, occurs at a significantly higher mean contact pressure, $P_{\mathrm{m}}$, than predicted by continuum crystal plasticity. For small indentation depths, the mean contact pressure increases rapidly with indentation depth $u$ but eventually a plateau value of $P_{\mathrm{m}}$ is reached, albeit one that is significantly higher than that obtained in the crystal plasticity calculations. In the discrete dislocation plasticity calculations, attainment of the plateau is associated with bulk yield in the sense that plastic deformation is not confined to the vicinity of the contact region, as will be discussed in more detail in the subsequent section.

The crystal with contact fraction $a / w=1 / 6$ shows intermediate behavior: the yield point is much higher than the one according to continuum crystal plasticity, as is typical of widely spaced contacts; on the other hand, the plateau value of $P_{\mathrm{m}}$ is attained shortly after yielding, which is characteristic of closely spaced contacts.

When perfectly sticking conditions prevail (Figure 2b), the unit cell cannot expand because the indenter is rigid and this precludes bulk yielding. The continuum crystal plasticity curves underestimate (compared to discrete dislocation plasticity) the hardening at contact fractions greater than $1 / 1.3$. Crystals with $a / w \leq 1 / 3$ deform only in the vicinity of the contact surface by a wedging mechanism similar to what would be described by slip line field theory: the material is squeezed out from below the indenter to pile up beside the indenter (as discussed in more detail in the subsequent section). When the spacing between indents is smaller than twice the contact size, this mechanism is hindered and plastic flow is inhibited. On the other hand, the unit cell can expand if the contacts are frictionless, thus allowing for the plastic flow to occur even for very closely spaced contacts (i.e. $a / w=1 / 1.5$ ). For sticking contacts, the discrete dislocation plasticity calculations show a similar trend for all contact fractions: the mean contact pressure, $P_{\mathrm{m}}$, is greater than that predicted by 
conventional crystal plasticity and a significantly higher strain hardening occurs. The only exception to the general trend is again the crystal with $a / w=1 / 1.5$, for which crystal plasticity gives an elastic response and discrete dislocation plasticity predicts little local stress relaxation.

Comparison of Figures $2 \mathrm{a}$ and $2 \mathrm{~b}$ reveals that the value $P_{\mathrm{m}}$, at a given indentation depth, predicted by conventional crystal plasticity, is not particularly sensitive to whether the contacts are frictionless or perfectly sticking for contact fractions greater than $1 / 6$. In contrast, the discrete dislocation plasticity predictions of $P_{\mathrm{m}}$ are increasingly sensitive to the friction condition with increasing contact fraction. With a contact size $a=1 \mu \mathrm{m}$ and for small contact fractions the discrete dislocation plasticity predictions are basically independent of the friction condition when the indentation depth is small. The difference between the two friction conditions appears at indentation depths greater than $u \simeq 0.11 \mu \mathrm{m}$. Then, bulk yielding occurs for the frictionless contacts, and the value of $P_{\mathrm{m}}$ reaches a plateau contacts, whereas $P_{\mathrm{m}}$ continues to increase for the perfectly sticking contacts.

\subsection{Deformation patterns}

The predicted distortion of the crystals at $u=0.1 \mu \mathrm{m}$ is shown in terms of deformed meshes for three unit cells for the two contact fractions $a / w=1 / 3$ and $a / w=1 / 12$ under frictionless contacts. The deformed meshes for sticking contacts at the same indentation depth are very similar to those for frictionless contacts and therefore not shown. For discrete dislocation plasticity simulations the deformed meshes are plotted in Figure 3. The crystal with the larger contact fraction, $a / w=1 / 3$ in Figure $3 \mathrm{a}$, has undergone a much larger bulk compression than the crystal with $a / w=1 / 12$. With more widely spaced contacts, $a / w=1 / 12$ in Figure $3 b$, plastic deformation is mainly confined to the contact vicinity, with material sink-in at the free surface close to the contact. A convenient measure for the extent of plasticity is the average strain in the $x_{1}$-direction, $\varepsilon_{11}=V / w$ : small values indicate localized plasticity, while bulk plasticity gives rise to large values. For the results in Figure 3, $\varepsilon_{11}$ is largest for $a / w=1 / 3\left(\varepsilon_{11}(a / w=1 / 3)=0.0047\right)$, while $\varepsilon_{11}(a / w=1 / 12)=0.0013$.

For deformed meshes obtained from the conventional crystal plasticity computations shown in Figure 4, the average lateral strain is also larger for $a / w=1 / 12$ $\left(\varepsilon_{11}(a / w=1 / 3)=0.0027\right)$ than for the more closely spaced contacts $\left(\varepsilon_{11}(a /\right.$ $w=1 / 12)=0.00021)$. However, when compared to the discrete dislocation plasticity results, the value of the average strain $\varepsilon_{11}$ is smaller for all contact fractions.

This difference in average strain between conventional crystal plasticity and discrete dislocation plasticity is related to the way in which the development of a density of geometrically necessary dislocations in the contact vicinity affects the material behavior. In discrete dislocation plasticity, geometrically necessary dislocations induce a size-dependent increase of the flow strength in the calculations. The increased strength promotes sink-in rather than pile-up, as opposed to the size-independent response according to conventional plasticity. Pile-ups and sink-ins are more pronounced when contacts are more isolated and the deformation more localized. If we define the pile-up height as $\max \left(\left(u_{2}\left(x_{1}, h\right)-u_{2}(w /\right.\right.$ $2, h)$ ) along the indented surface, we find that in the crystal plasticity predictions, the pileup height ranges from $4 \mathrm{~nm}$ for $a / w=1 / 3$ to $26 \mathrm{~nm}$ for $a / w=1 / 12$. For closely spaced contacts, the pile-ups are generated by the wedging mechanism of neighboring contacts 

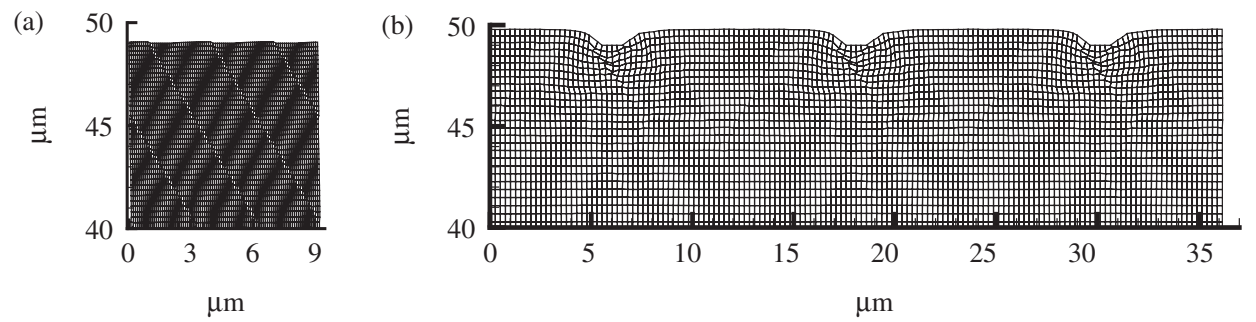

Figure 3. Deformed meshes obtained from discrete dislocation plasticity for contacts with $a=1 \mu \mathrm{m}$ and (a) $a / w=1 / 3$ and (b) $a / w=1 / 12$ at $u=0.1 \mu \mathrm{m}$. Displacements are magnified by a factor of 10 .
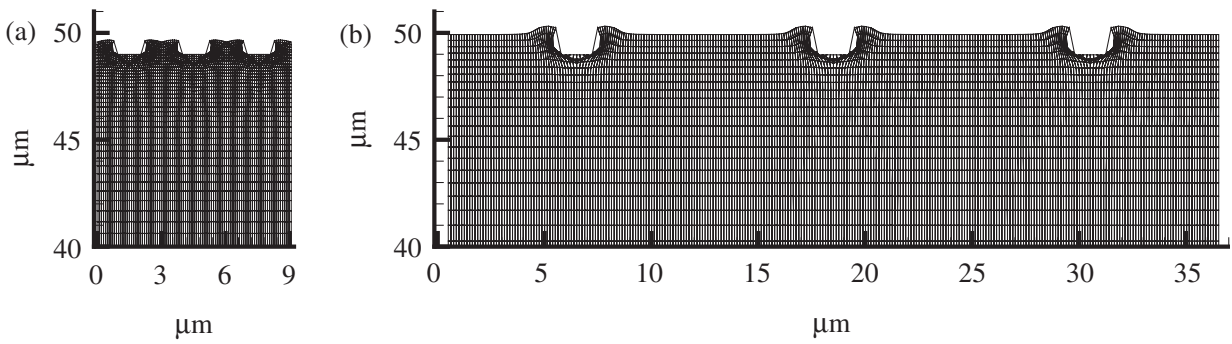

Figure 4. Deformed mesh according to conventional crystal plasticity for (a) $a / w=1 / 3$ and (b) $a / w=1 / 12$ at $u=0.1 \mu \mathrm{m}$. Displacements are magnified by a factor of 10 .

overlap (see Figure 4a). For such contacts, it is impossible to identify a non piled-up region and, therefore, to give a measure of the material pile-up that compares with the measure obtained for more separated contacts.

The deformation modes can also be visualized through the distribution of the lattice rotation, as given in Figure 5. The lattice rotation (positive in the counter-clockwise direction) is computed from the discrete dislocation plasticity displacement field according to

$$
\Omega=\frac{1}{2}\left[\left(\hat{u}_{2,1}+\tilde{u}_{2,1}\right)-\left(\hat{u}_{1,2}+\tilde{u}_{1,2}\right)\right]
$$

where the spatial differentiation of $\tilde{\mathrm{u}}_{i}$ (denoted by ${ }_{j}$ ) is performed analytically. The lattice rotations for rather isolated contacts, Figure $5 \mathrm{~b}$, are concentrated in the regions next to the contacts where sink-in takes place and the rotations are largely geometrically necessary; directly below the indenter the rotation is much smaller. Inside the sink-in regions, the lattice rotation is similar to that found in discrete dislocation simulations of wedge indentation [4], both in terms of the distribution and the magnitude (for a given indentation depth). For closely spaced contacts, Figure 5a, the distribution is very different: rotation is confined to shear bands that extend well into the crystal.

\subsection{Internal stress state}

In this section we present stress distributions and, for the discrete dislocation calculations, dislocation distributions at an indentation depth of $u=0.1 \mu \mathrm{m}$. Figure 6 presents the stress distributions, computed using discrete dislocation plasticity in crystals with frictionless 

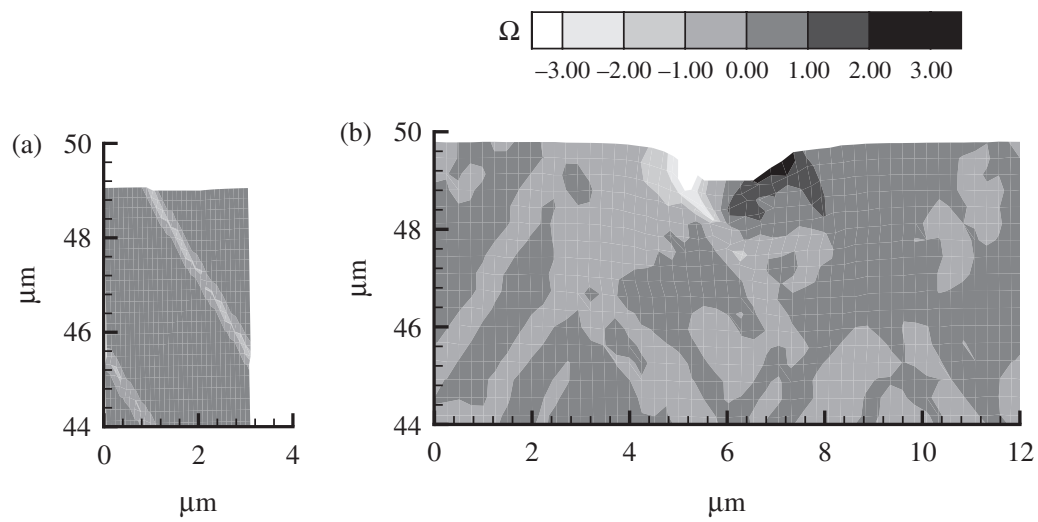

Figure 5. Lattice rotation $\Omega$ (in degrees) according to discrete dislocation plasticity at $u=0.1 \mu \mathrm{m}$ for contact fractions of (a) $a / w=1 / 3$ and (b) $1 / 12$ under frictionless conditions.
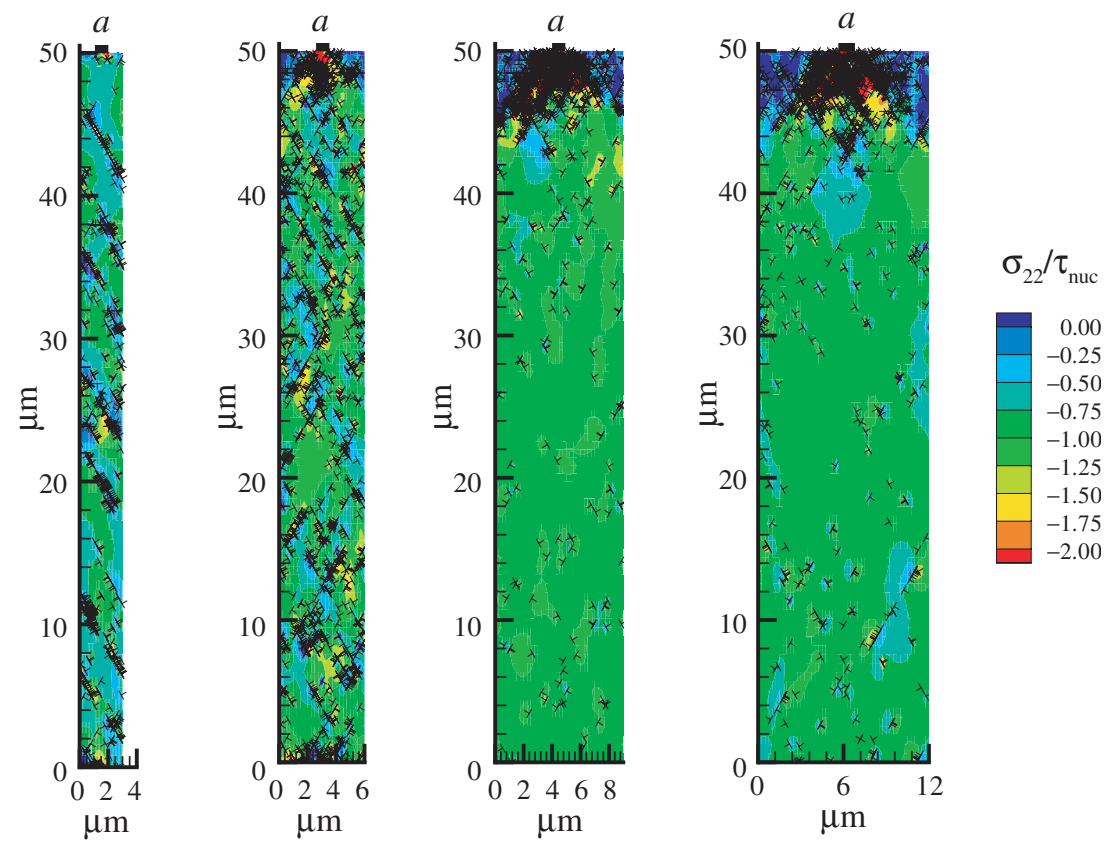

Figure 6. (Color online). Combined dislocation and stress $\left(\sigma_{22}\right)$ distributions at $u=0.1 \mu \mathrm{m}$ for frictionless contacts with contact size $a=1 \mu \mathrm{m}$ and contact fractions: (a) $a / w=1 / 3$, (b) $a / w=1 / 6$ and (c) $a / w=1 / 9$ and (d) $a / w=1 / 12$.

contacts (as in Figure 2a) and the corresponding dislocation structures. The stress $\sigma_{22}$ is normalized by the average nucleation strength of the dislocation sources, $\tau_{\text {nuc }}=50 \mathrm{MPa}$. At $u=0.1 \mu \mathrm{m}$ several dislocations are present far away from the contacts for all contact fractions shown in Figure 6. However, while the dislocation density is almost independent of $x_{2}$ for $a / w=1 / 3$ and $a / w=1 / 6$ (Figures $6 \mathrm{a}, \mathrm{b}$ ), the dislocations are concentrated in 
a region near the contact for $a / w=1 / 9$ and $a / w=1 / 12$ (Figures $6 \mathrm{c}, \mathrm{d}$ ). For the largest contact fraction, $a / w=1 / 3$, plastic deformation occurs primarily on one set of slip planes $\left(\phi=120^{\circ}\right)$ throughout the unit cell. Plasticity underneath the well-separated contacts, $a / w=1 / 9$ and $a / w=1 / 12$, is mainly confined to the contact region. Contacts with $a / w=1 / 6$, as noted in the previous section, appear to have an intermediate behavior: combining a relatively high dislocation density in the bulk with an increased dislocation density in the contact vicinity.

Figure 7 shows stress and dislocation distributions in a region near the contact surface for the contacts with contact fractions $a / w=1 / 3$ and $1 / 12$. A highly stressed zone can be seen under the indenter in the crystal with $a / w=1 / 12$, while there is only a very small highly stressed zone under one of the indenter corners when $a / w=1 / 3$. The crystal under the more widely spaced contacts, Figure $7 \mathrm{~b}$, shows a highly stressed zone that starts underneath the contact and propagates for several micrometers inside the crystal. The width of the region where stresses are at least $100 \mathrm{MPa}$ in magnitude is quite extended, up to eight times larger than the contact area. The dislocation activity on intersecting slip planes leads to dislocation junctions at the intersection, which act as obstacles for dislocation glide. When a dislocation junction forms at the intersection between slip planes, other slip planes are likely to become active to accommodate the deformation at a higher imposed load.

Figure 8 shows $\sigma_{22}$ stress distributions at the same relative indentation depth obtained from the continuum crystal plasticity calculations (here stress is normalized by the slip system strength $\tau_{0}=20 \mathrm{MPa}$ ). The crystal with the largest contact fraction, $a / w=1 / 3$ (Figure 8), has the highest stress, $\sigma_{22}=40 \mathrm{MPa}$, in the crystal bulk, which corresponds to bulk yield. This is the same average stress obtained from the discrete dislocation plasticity simulations. The crystals with smaller contact fractions exhibit high stresses only in the vicinity of the contact, but the highly stressed regions are smaller than in the discrete dislocation plasticity simulations shown in Figures 6 and 7.
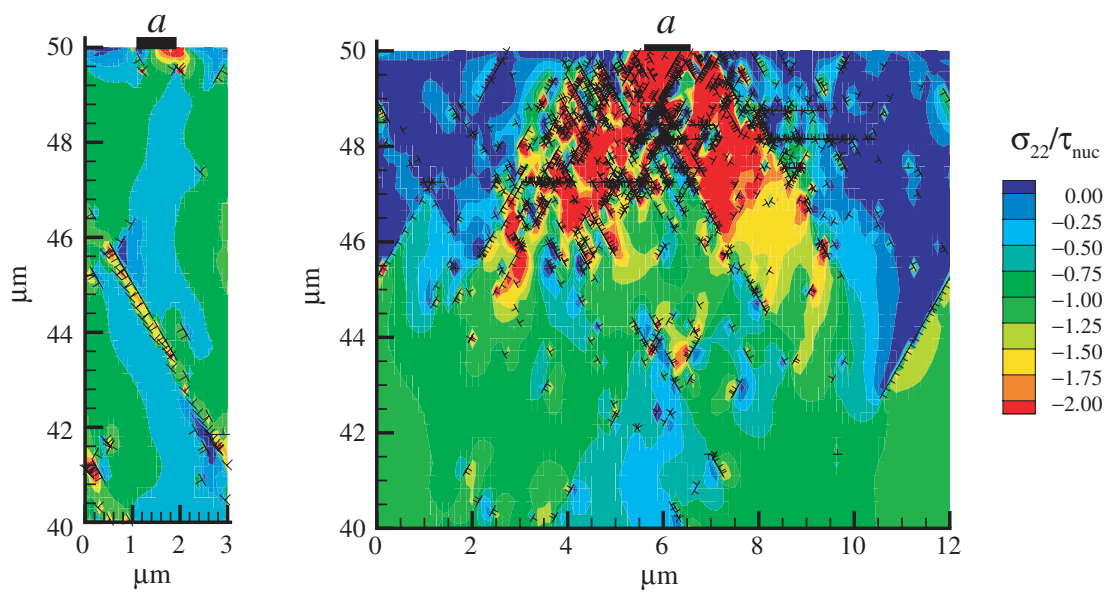

Figure 7. (Color online). Zoomed-in distribution of $\sigma_{22}$ and dislocation structure for the contacts with (a) $a / w=1 / 3$ and (b) $a / w=1 / 12$ in Figure 6. 
The discrete dislocation plasticity results for perfectly sticking contacts, Figure 9, differ from the corresponding results for frictionless contacts (Figure 6), especially for large contact fractions; i.e. $a / w=1 / 3$ and $a / w=1 / 6$ in Figures $9 \mathrm{a}, \mathrm{b}$. For frictionless contacts, the average value of $\sigma_{11}$ in the unit cell is zero. On the other hand, with perfectly sticking contacts the lateral deformation of the unit cell is constrained, thus building up a negative lateral stress. This results in a stress state that is largely hydrostatic stress away from the contact surface, thus inhibiting dislocation activity.

\section{Size effect}

In this section, results are presented showing the effect of varying all geometric lengths; i.e. the contact size $a$, the contact spacing $w$ and the crystal height $h$. Material lengths, such as the Burgers vector and the average source and obstacle spacing, are kept constant.

To make contact with Nicola et al. [13], we first consider cases with fixed contact fraction $a / w=1 / 9$ and with the contact size $a$ ranging from $0.125 \mu \mathrm{m}$ to $1 \mu \mathrm{m}$. Plots of mean contact pressure $P_{\mathrm{m}}$ versus indentation depth are shown in Figure 10. The indentation depth $u$ is normalized by the contact spacing so that the responses are self-similar when the material behavior is size independent, as in the elastic regime, and as predicted by continuum crystal plasticity. By contrast, the discrete dislocation plasticity curves reveal a size dependent response, with smaller contacts being harder than larger contacts, both for perfectly sticking and frictionless contacts. Results are also shown in Figure 10 for crystal plasticity simulations using a hardening coefficient $h_{0} / \mu=2.5 \times 10^{-3}$, which is three orders of magnitude larger than the value $h_{0} / \mu=2.5 \times 10^{-6}$ used in all other calculations. With this hardening coefficient, the crystal plasticity predictions give a better fit to the discrete dislocation plasticity results for this particular contact fraction, but the size effect obviously cannot be captured.

From Figure 2, with the contact size $a$ fixed at $1 \mu \mathrm{m}$, it was found that for small indentation depths $u$ the friction condition had an effect on the indentation pressure $P_{\mathrm{m}}$ only for closely spaced contacts. For a contact fraction of $a / w=1 / 9$ the friction condition

(a)

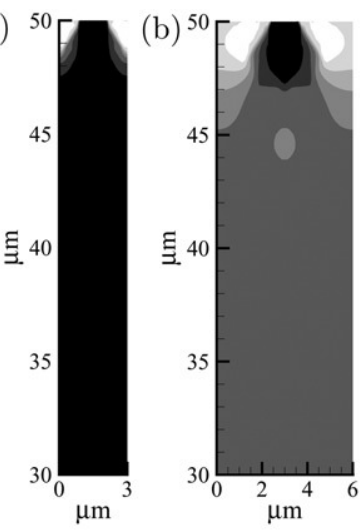

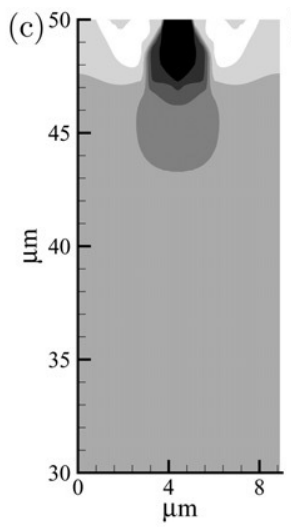

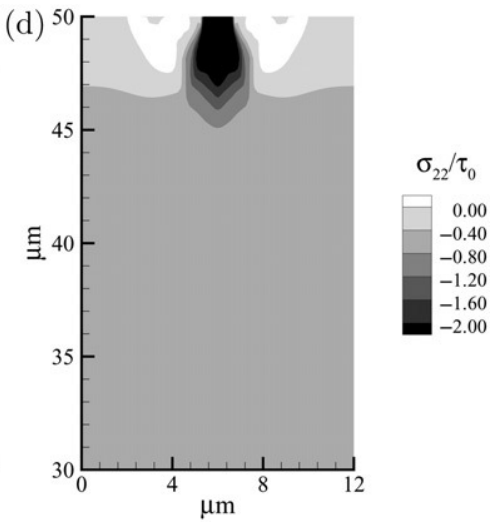

Figure 8. (Color online). Continuum crystal plasticity predictions of the distribution of $\sigma_{22}$ at $u=0.1 \mu \mathrm{m}$ for crystals with $a=1 \mu \mathrm{m}$ and (a) $a / w=1 / 3$, (b) $a / w=1 / 6$, (c) $a / w=1 / 9$ and (d) $a / w=1 / 12$. 

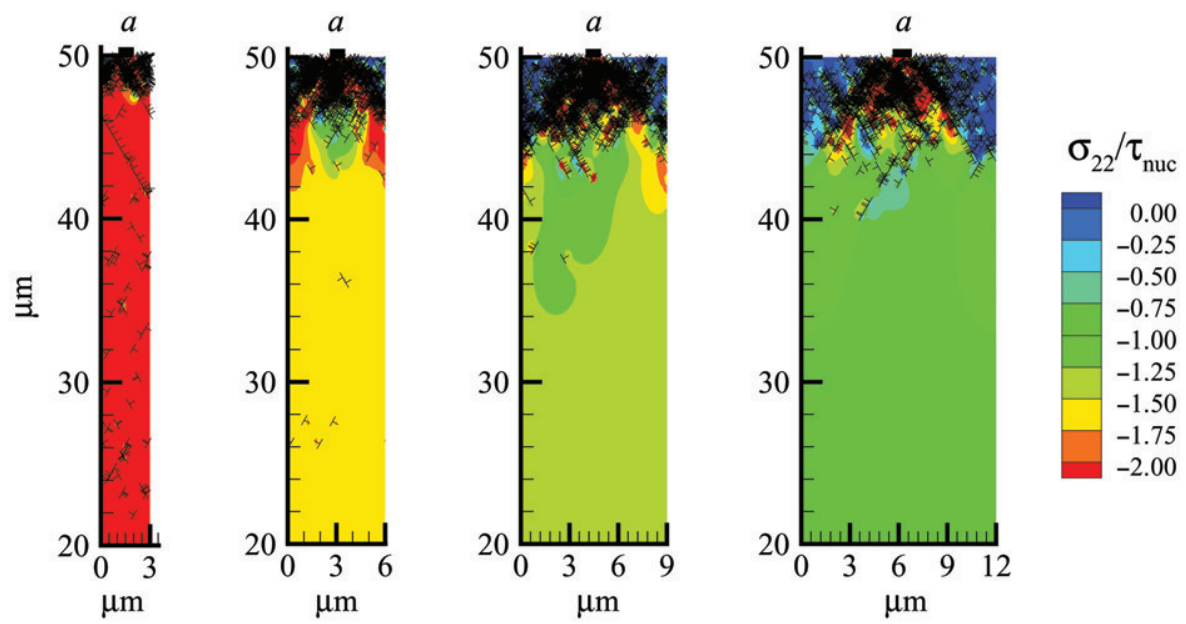

Figure 9. (Color online). Discrete dislocation plasticity predictions for the distribution of $\sigma_{22}$ and the dislocation structure at $u=0.1 \mu \mathrm{m}$ for crystals having contact size $a=1 \mu \mathrm{m}$ and contact fraction (a) $a / w=1 / 3$, (b) $a / w=1 / 6$ and (c) $a / w=1 / 9$ and (d) $a / w=1 / 12$.
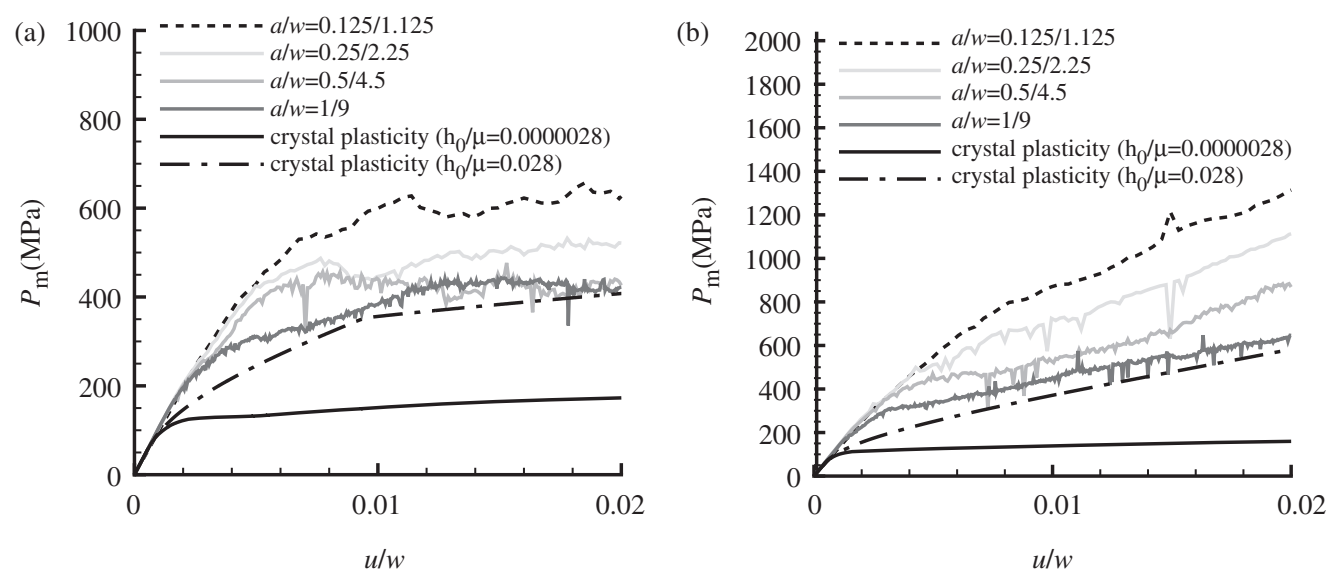

Figure 10. Mean contact pressure, $P_{\mathrm{m}}$, versus indentation depth, $u$, normalized by cell width for crystals with the same value of the contact fraction $a / w=1 / 9$ and contact size ranging from $a=0.125 \mu \mathrm{m}$ to $1 \mu \mathrm{m}$. (a) Frictionless contacts. (b) Perfectly sticking contacts.

did not affect the value of $P_{\mathrm{m}}$ for small indentation depths. However, the results in Figure 10 show that friction conditions do matter for a contact fraction $1 / 9$ when the contacts are sufficiently small, even at small indentation depth $u$. When the contact size is $a=0.5 \mu \mathrm{m}$, the mean contact pressure at $u / w=0.02$ is $400 \mathrm{MPa}$ for frictionless contacts and $800 \mathrm{MPa}$ for sticking contacts.

The crystal under frictionless contacts with $a=0.5 \mu \mathrm{m}$ and $w=4.5 \mu \mathrm{m}$ deforms by bulk yield according to the same mechanism, typical of closely spaced, but larger contacts. Thus, at the micron scale, the contact fraction alone is not sufficient to establish the deformation mode of a crystal under multi-asperity contacts; the actual size of the contacts matters (and of course the material properties). The smaller the contact size, the smaller 

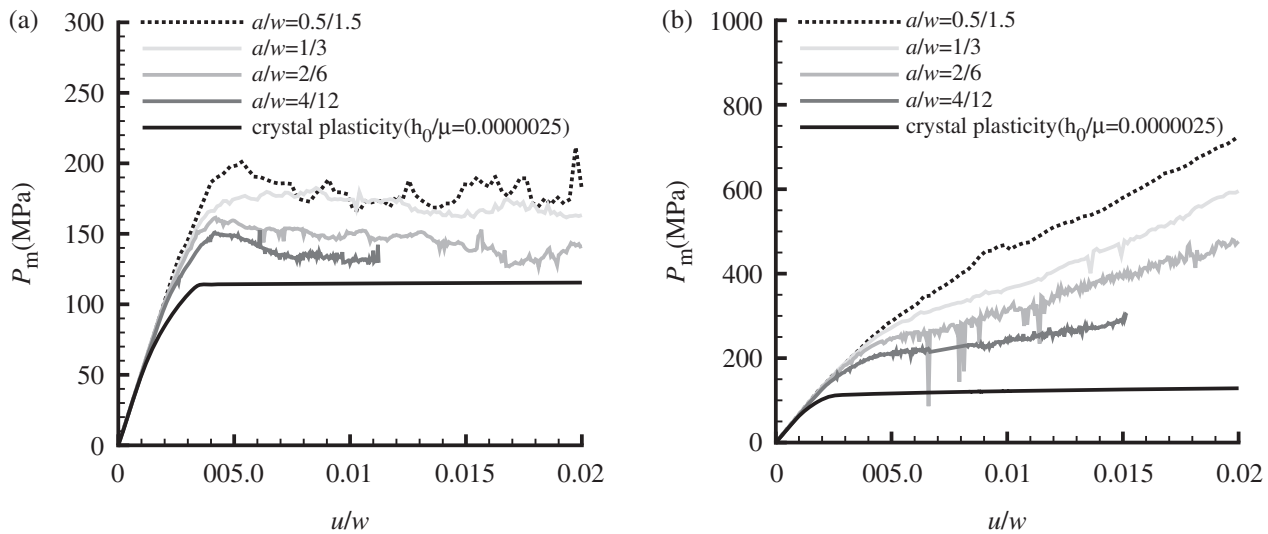

Figure 11. Mean contact pressure, $P_{\mathrm{m}}$, versus indentation depth normalized by cell width for crystals with the same value of the contact fraction $a / w=1 / 3$ and contact size ranging from $a=1.5 \mu \mathrm{m}$ to $4 \mu \mathrm{m}$. (a) Frictionless contacts. (b) Perfectly sticking contacts.

the contact fraction needed for the contact to behave as isolated; i.e. having the following characteristics: (i) localized deformations; (ii) overall hardening in the $P_{\mathrm{m}}$ versus $u$ response; and (iii) the response independent of friction conditions.

Figure 11 shows corresponding results for closely spaced contacts, $a / w=1 / 3$, with a contact size ranging from $a=0.5 \mu \mathrm{m}$ to $4 \mu \mathrm{m}$. Here too, discrete dislocation plasticity gives a size dependent response. With frictionless contacts, Figure 11a, the size dependence is mainly in the yield point, with crystals under smaller contacts yielding later than the ones under large contacts. This size effect can be attributed to limited source availability: if there are no sources available in the region where stresses are sufficiently high, plastic flow will not take place. Once yielding occurs, dislocation activity appears to be relatively independent of contact size and the evolution of the mean contact pressure is essentially size independent. In contrast, for perfectly sticking contacts, Figure $11 \mathrm{~b}$, the evolution of the mean contact pressure after yielding is influenced by contact size; for a contact size of $0.5 \mu \mathrm{m}$ the crystal exhibits nearly elastic behavior.

The size effects in Figures 10 and 11 are not captured by conventional crystal plasticity. Also, currently available strain gradient plasticity theories cannot capture all size effects seen in the discrete dislocation plasticity calculations. Indentation analyses based on sizedependent phenomenological plasticity theories related to the concept of geometrically necessary dislocations, as for example in [29-31], predict a size effect arising from plastic strain gradients. However, a size dependent onset of yielding related to the discreteness of dislocation sources (source limited plasticity) is not modeled in such analyses. Models, based on statistical mechanics considerations that are currently under development by Groma, Zaiser and others [32-34], appear promising for capturing both gradient related and source limitation related size depenendence.

\section{Conclusions}

We have presented a numerical study of plane strain multi-asperity contact between a rigid indenter and a single crystal. Conventional crystal plasticity and discrete dislocation 
plasticity computations have been carried out for the limiting cases of perfectly frictionless and perfectly sticking contacts. The results show the following general features:

- According to conventional crystal plasticity, except for very closely spaced contacts, deformation of a single crystal under equispaced asperities occurs by a wedging mechanism of the type described by slip line theory with plastic flow confined to a region beneath the contacts.

- The evolution of the mean contact pressure is nearly independent of the friction condition. Friction plays an important role only when the spacing between individual contacts is smaller than twice the contact size in which case the wedging mechanism is suppressed under sticking conditions.

- The wedging mechanism leads to material pile-up around the contacts for all contact fractions $a / w$ analyzed.

- Discrete dislocation plasticity predicts a size effect for micron scale contacts.

- The size effect originates from: (i) geometrically necessary dislocations; and (ii) the limited availability of dislocation sources beneath the micron scale contacts. The size effect is more pronounced for perfectly sticking contacts and when the contact fraction $a / w$ is small.

- For small contact fraction $a / w$ and sufficiently large contact size $a$, discrete dislocation plasticity predicts that:

- The deformation is localized underneath the contacts (as for conventional continuum crystal plasticity) but material sink-in rather than pile-up occurs around the asperities.

- The mean contact pressure increases with indentation depth at a larger rate than predicted by crystal plasticity.

- The response is essentially independent of friction conditions.

- When contacts are sufficiently close to each other (large contact fraction $a / w$ ):

- Indentation induces plastic flow throughout the crystal for frictionless contacts.

- Plastic flow is largely suppressed for perfectly sticking contacts.

\section{Acknowledgements}

L.N., A.F.B., K.-S. K. and A.N. gratefully acknowledge support from the Materials Research Science and Engineering Center at Brown University (NSF Grant DMR-0520651).

\section{References}

[1] M.C. Fivel, C.F. Robertson, G.R. Canova et al., Acta Materialia 46 (1998) p.6183.

[2] H.G.M. Kreuzer and R. Pippan, Mater. Sci. Eng. A 387 (2004) p.254.

[3] A. Widjaja, A. Needleman and E. van der Giessen, Model. Simul. Mater. Sci. Eng. 15 (2007) p.121.

[4] D.S. Balint, V.S. Deshpande, A. Needleman et al., J. Mech. Phys. Solids 54 (2006) p.2281. 
[5] Y.F. Gao, A.F. Bower, K.S. Kim et al., Wear 261 (2006) p.145.

[6] L. Pei, S. Hyun, J.F. Molinari et al., J. Mech. Phys. Solids 53 (2005) p.2385.

[7] Y.F. Gao and A.F. Bower, Proc. Royal Soc. London, Series A 462 (2006) p.319.

[8] J.G. Swadener, E.P. George and G.M. Pharr, J. Mech. Phys. Solids 50 (2002) p.681.

[9] J. Wang, J. Lian, J.R. Greer et al., Acta Materialia 54 (15) (2006) p.3973.

[10] J. Wang, K.-S. Kim, to be submitted to Appl. Phys. Lett.

[11] Y. Zhao and L. Chang, ASME J. Tribology 123 (2001) p.857.

[12] M. Ciavarella, V. Delfine and V. Demelio, Wear 261 (2006) p.556.

[13] L. Nicola, A.F. Bower, K.S. Kim et al., J. Mech. Phys. Solids 55 (2007) p.1120.

[14] J. Wang, P. Shrotriya and K.-S. Kim, Experim. Mech. 46 (2006) p.39.

[15] E. Van der Giessen and A. Needleman, Model. Simul. Mater. Sci. Eng. 3 (1995) p.689.

[16] A.C. Chng, M.P. O’Day, W.A. Curtin et al., Acta Materialia 54 (2006) p.1017.

[17] V.S. Deshpande, A. Needleman and E. Van der Giessen, Acta Materialia 51 (2003) p.4637.

[18] S.M. Keralavarma and A.A. Benzerga, Model. Simul. Mater. Sci. Eng. 15 (2007) p.239.

[19] J. Senger, D. Weygand, P. Gumbsch et al., Scripta Materialia 58 (2008) p.587.

[20] S.B. Biner, Phil. Mag. 83 (2003) p.3677.

[21] S.J. Noronha and N.M. Ghoniem, Metal. Mater. Trans. A Phys. Metal. Mater. Sci. 73A (2006) p.539.

[22] A.A. Benzerga, Y. Bréchet, A. Needleman et al., Model. Simul. Mater. Sci. Eng. 12 (2004) p.159.

[23] L. Nicola, E. Van der Giessen and A. Needleman, J. Appl. Phys. 93 (2003) p.5920.

[24] L. Nicola, Y. Xiang, J.J. Vlassak et al., J. Mech. Phys. Solids 54 (2006) p.2089.

[25] J.Y. Shu, N.A. Fleck, E. Van der Giessen et al., J. Mech. Phys. Solids 49 (2001) p.1361.

[26] L.P. Kubin, G.R. Canova, M. Condat et al., Solid State Phenom. 23 (1992) p.455.

[27] V.S. Deshpande, A. Needleman and E. Van der Giessen, Disloc. Solids 13 (2007) p.1.

[28] D. Peirce, R.J. Asaro and A. Needleman, Acta Metallurgica 31 (1983) p.1951.

[29] M.R. Begley and J.W. Hutchinson, J. Mech. Phys. Solids 46 (1998) p.2049.

[30] Y. Wei and J.W. Hutchinson, J. Mech. Phys. Solids 51 (2003) p.2037.

[31] S. Qu, Y. Huang, G.M. Pharr et al., Internat. J. Plasticity 22 (2006) p.1265.

[32] I. Groma, F.F. Csikor and M. Zaiser, Acta Materialia 51 (2003) p.1271.

[33] S. Yefimov, I. Groma and E. Van der Giessen, Model. Simul. Mater. Sci. Eng. 12 (2004) p.1069.

[34] S. Limkumnerd and E. Van der Giessen, J. Mech. Phys. Solids 56 (2008), p.3304. 